\title{
GENERALIZED ALEXANDROV-TOPONOGOV THEOREMS FOR RADIALLY CURVED MANIFOLDS AND THEIR APPLICATIONS
}

\author{
Hyunjin LEE \\ (Received 27 September 2004)
}

\begin{abstract}
We investigate the topology of complete Riemannian manifolds with the radial curvature at the base manifold bounded below by those of model surfaces. Here, model surfaces are generalized surfaces of revolution and warped product models which are diffeomorphic to $\mathbf{S}^{n}$.
\end{abstract}

\section{Introduction}

The Alexandrov-Toponogov comparison theorems play an essential role for the investigation of curvature and topology of Riemannian manifolds. Many attempts have been made to extend the reference spaces to those with non-constant curvature, for instance, Abresch [1, 2], Elerath [3], Greene and Wu [4], and Machigashira [7, 8], etc. They mainly deal with Hadamard surfaces of revolution whose Gaussian curvature has special restrictions.

We discuss two kinds of reference spaces. One of them has the rotationally symmetric metric around the base point $\tilde{o}$ of a reference surface $\tilde{M}$, where the cut locus $C(\tilde{o})$ to $\tilde{o}$ is either an empty set (when $\tilde{M}$ is diffeomorphic to $\mathbb{R}^{n}$ ) or $C(\tilde{o})$ coincides with the first conjugate locus which is a single point, say $\tilde{o}_{1} \in \tilde{M}$ (when $\tilde{M}$ is diffeomorphic to $\left.\mathbf{S}^{n}\right)$. Such a $(\tilde{M}, \tilde{o})$ is called a model surface of revolution.

The other reference space is a warped product model $(\tilde{M}, N)$, where $\tilde{M}:=$ $\left(-\tilde{\ell}_{-}, \tilde{\ell}_{+}\right) \times N$ admits the metric expressed by the normal exponential map along the base manifold $N$ as (the precise definition is given later)

$$
d s_{\widetilde{M}}^{2}=d t^{2}+f^{2}(t) d s_{N}^{2}(x), \quad(t, x) \in\left(-\tilde{\ell}_{-}, \tilde{\ell}_{+}\right) \times N .
$$

2000 Mathematics Subject Classification: Primary 53C20, 53C21.

Keywords and Phrases: Riemannian manifold; warped product model; radial curvature; Clairaut relation; Toponogov theorem. 
Here $\tilde{\ell}_{ \pm} \leq \infty$ are positive constants. $N$ is identified with $\{0\} \times N$ and a compact connected totally geodesic hypersurface of $\widetilde{M}$ (and is called the base manifold of $\widetilde{M}$ ). The warping function $f:\left(-\tilde{\ell}_{-}, \tilde{\ell}_{+}\right) \rightarrow \mathbb{R}$ is positive smooth satisfying the Jacobi equation:

$$
f^{\prime \prime}(t)+K(t) f(t)=0, \quad f(0)=1, \quad f^{\prime}(0)=0, \quad t \in\left(-\tilde{\ell}_{-}, \tilde{\ell}_{+}\right) .
$$

The function $K:\left(-\tilde{\ell}_{-}, \tilde{\ell}_{+}\right) \rightarrow \mathbb{R}$ is called the radial curvature function of a warped product model $(\tilde{M}, N)$. Note that the cut locus $C(N)$ to $N$ is either an empty set (when $\left.\tilde{\ell}_{-}=\tilde{\ell}_{+}=\infty\right)$ or $C(N)$ consists of at most two points. $\tilde{M}$ is diffeomorphic to $\mathbb{R} \times N$ if $C(N)=\emptyset$. Otherwise, $C(N)$ coincides with the first focal locus to $N$. Moreover, $\widetilde{M}$ is diffeomorphic to either $\mathbb{R}^{n}$ if and only if $C(N)$ consists of a single point (i.e. one of the two constants $\tilde{\ell}_{ \pm}$is finite) or else $\mathbf{S}^{n}$ if and only if $C(N)$ consists of two points (i.e. both $\tilde{\ell}_{-}$and $\tilde{\ell}_{+}$are finite). We prove topological sphere theorems by using the model spaces with non-constant curvature. For simplicity, we only discuss warped product models which are diffeomorphic to $\mathbf{S}^{n}$. We may understand that every compact model surface of revolution is a compact warped product model by taking a suitable level hypersurface at which $f$ takes a positive critical value.

Let $M$ be a connected complete Riemannian $n$-manifold with a base point at $o$ or with a compact connected $(n-1)$-dimensional totally geodesic hypersurface $N$. Let $\gamma:[0, a) \rightarrow M, a \leq \infty$ be a geodesic with $\gamma(0) \in N, \dot{\gamma}(0) \in N^{\perp}$ and $d(\gamma(t), N)=t$ for all $t \in[0, a)$. Here $N^{\perp}$ is the normal bundle of $N$. Such a $\gamma$ is called a minimizing geodesic from $N$. Let $(\tilde{M}, N)$ (or $(\tilde{M}, \tilde{o}))$ be a warped product model and $K:\left(-\tilde{\ell}_{-}, \tilde{\ell}_{+}\right) \rightarrow \mathbb{R}$ the radial curvature function of $(\tilde{M}, N)$. We say that the radial curvature of $(M, N)$ is bounded below by $K$ if and only if along every minimizing geodesic $\gamma:[0, a) \rightarrow M$ from $N$ the sectional curvature of $M$ satisfies

$$
K_{M}(\dot{\gamma}(t), X) \geq K(t), \quad t \in[0, a), \quad X \in M_{\gamma(t)}, \quad X \perp \dot{\gamma}(t)
$$

Here, the plane spanned by $\dot{\gamma}(t)$ and $X$ is a radial plane and $K_{M}(\dot{\gamma}(t), X)$ is a radial curvature of $(M, N)$. The radial curvature of $(M, o)$ is similarly defined when $N=\{o\}$. We say that $(M, o)$ is referred to $(\tilde{M}, \tilde{o})$ if and only if $(1.3)$ is satisfied along every minimizing geodesic emanating from $o$. By means of the axiom of plane, we only need to consider a model surface of revolution which is isometrically and totally geodesically embedded into $\widetilde{M}$ via the exponential map at $\tilde{o}$ restricted to a twodimensional subspace of $\tilde{M}_{\tilde{o}}$. Let $(\tilde{M}, \tilde{o})$ be a compact model surface of revolution with the metric (1.1) for $N=\mathbf{S}^{n-1}$ and $K:[0, \tilde{\ell}] \rightarrow \mathbb{R}$ its radial curvature function of $(\tilde{M}, \tilde{o})$. Let $3 \varepsilon_{1}(\tilde{M}):=\delta(\tilde{M})$ be the convexity radius of $\tilde{M}$ and let $\varepsilon(\tilde{M})$ be a 
positive constant such that

$$
\varepsilon(\tilde{M}):=\sup \left\{\varepsilon>0 \mid \min \{f(2 \varepsilon), f(\tilde{\ell}-2 \varepsilon)\}=\min _{2 \varepsilon \leq t \leq \tilde{\ell}-2 \varepsilon} f(t)\right\}
$$

and $\varepsilon(\tilde{M}) \leq \varepsilon_{1}(\tilde{M})$. Let $o^{*}$ be the point furthest from $\tilde{o}$ such that $C(\tilde{o})=\left\{o^{*}\right\}$, $C\left(o^{*}\right)=\{\tilde{o}\}$ and $d\left(\tilde{o}, o^{*}\right)=\tilde{\ell}$. Let $A \subset M$ be a compact set and let $\rho_{A}: M \backslash A \rightarrow \mathbb{R}$ be the distance function to $A$ :

$$
\rho_{A}(x):=d(x, A), \quad x \in M \backslash A .
$$

With this notation our first result is stated as follows.

THEOREM A. Let $(\tilde{M}, \tilde{o})$ be a model surface of revolution with $\tilde{\ell}<\infty$. Let $(M, o)$ be referred to $(\tilde{M}, \tilde{o})$. Then $M$ is homeomorphic to $\mathbf{S}^{n}$ if $\delta(o) \geq 3 \varepsilon_{1}(\tilde{M})$, $\max \rho_{o}>\tilde{\ell}-\varepsilon(\tilde{M})$ and $\operatorname{diam}(M) \leq \tilde{\ell}$.

Let $(\tilde{M}, N)$ be a warped product model diffeomorphic to $\mathbf{S}^{n}$. We say that $(M, N)$ is referred to $(\widetilde{M}, N)$ (or the reference space of $(M, N)$ is $(\tilde{M}, N)$ ) if and only if $N$ is totally geodesic and (1.3) is satisfied along every minimizing geodesic from $N$.

Let $(M, N)$ be referred to a warped product model $(\tilde{M}, N)$ diffeomorphic to $\mathbf{S}^{n}$, and $M \backslash N=M_{+} \cup M_{-}, \tilde{M} \backslash N=\widetilde{M}_{+} \cup \tilde{M}_{-}$(disjoint union). Set

$$
\ell_{ \pm}:=\max _{x \in M_{ \pm}}\left|d_{M}(N, x)\right|, \quad \tilde{\ell}_{ \pm}:=\max _{\tilde{x} \in \widetilde{M}_{ \pm}}\left|d_{\widetilde{M}}(N, \tilde{x})\right| .
$$

Here $d_{M}(N, x)$ is the oriented distance function and takes the values on $\left[-\ell_{-}, \ell_{+}\right]$. Moreover, put $3 \varepsilon_{1}(\tilde{M}):=\delta(\tilde{M})$. We choose $\eta_{ \pm}\left(\tilde{M}_{ \pm}\right)>0$ satisfying that

$$
\eta_{ \pm}\left(\tilde{M}_{ \pm}\right):=\sup \left\{\eta>0 \mid f\left(\tilde{\ell}_{ \pm}-\eta\right)=\min _{0 \leq t \leq \tilde{\ell}_{ \pm}-\eta} f(t)\right\}
$$

and

$$
\eta_{ \pm}\left(\tilde{M}_{ \pm}\right) \leq \delta(\tilde{M})
$$

From now on, we set $\tilde{p}_{ \pm} \in \tilde{M}_{ \pm}$are points furthest from $N$ and $d\left(N, \tilde{p}_{ \pm}\right)=\tilde{\ell}_{ \pm}$. With this notation we state our final result.

THEOREM B. Let $(M, N)$ be referred to warped product model $(\tilde{M}, N)$. Here $N=\mathbf{S}^{n-1}$. If $\ell_{ \pm} \geq \tilde{\ell}_{ \pm}-\eta_{ \pm}\left(\tilde{M}_{ \pm}\right)$then $M$ is homeomorphic to $\mathbf{S}^{n}$.

Theorem A extends the result by Kondo (see $[5,6])$. He has given a topological sphere theorem when the reference space is a compact von Mangoldt surface $(\widetilde{M}, \tilde{o})$ 
of revolution with a singular point at $\tilde{o}_{1}$. Here $\tilde{o}_{1}$ is the unique point furthest from $\tilde{o}$ such that $d\left(\tilde{o}, \tilde{o}_{1}\right)=\tilde{\ell}$ and the metric is expressed by

$$
d s_{\widetilde{M}}^{2}=d t^{2}+f^{2}(t) d \theta^{2}, \quad(t, \theta) \in(0, \tilde{\ell}) \times \mathbf{S}^{1}
$$

and the radial curvature function is monotone and non-increasing on $[0, \tilde{\ell}]$.

THEOREM 1.1. (Kondo $[\mathbf{5}, \mathbf{6}])$ Let $(M, o)$ be a compact pointed Riemannian $n$-manifold whose radial curvature at $o \in M$ is bounded below by $K:[0, \tilde{\ell}) \rightarrow \mathbb{R}$ for $\tilde{\ell}<\infty$. If $\rho$ is the first zero of $f^{\prime}$ on $(0, \tilde{\ell})$ and if

$$
\max \rho_{o}>\rho,
$$

then, there exists a sufficiently small $\varepsilon_{0}:=\varepsilon\left(\max \rho_{o}\right)>0$ such that if

$$
\max \rho_{o}>\tilde{\ell}-\varepsilon_{0}
$$

then $(M, o)$ is homeomorphic to a sphere $\mathbf{S}^{n}$.

Our proof requires the Toponogov comparison theorem for (generalized) narrow triangles where the reference space is a general model surface of revolution and warped product model.

Topological restrictions on radially curved manifolds have been obtained in $[\mathbf{1 , 2}$, 7-10, 13]. Basic tools used for the proofs of our theorems and the characterization of the general warped product models are stated in Section 2. We give the proofs of our results in Section 3 .

\section{Review of the known results}

We restrict to consider warped product models of the form $\tilde{M}=\left(-\tilde{\ell}_{-}, \tilde{\ell}_{+}\right) \times_{f} N$, where $\tilde{\ell}_{ \pm}<\infty$ and $N$ is the standard $(n-1)$-sphere and $f$ a warping function such that

$$
f\left(-\tilde{\ell}_{-}\right)=f\left(\tilde{\ell}_{+}\right)=0, \quad f^{\prime \prime}+K f=0, \quad f(0)=1, \quad f^{\prime}(0)=0 .
$$

The model surfaces of revolution are considered as the special warped product models. We may discuss the warped product models. Because our models do not have constant curvature, we cannot apply the spherical trigonometry. The Clairaut relation gives restrictions to the behavior of geodesics on models and plays an essential role for our study. The following proposition is valid for all the warped product models as stated in the classification [9]. 
Proposition 2.1. (The Clairaut relation for warped product models, MashikoShiohama [9]) Let $(\tilde{M}, N)$ be a warped product model with the metric (1.1). Let $\tilde{\gamma}: \mathbb{R} \rightarrow \widetilde{M}$ be a geodesic transversal to a meridian. If we set

$$
\alpha(s):=L\left(\tilde{\gamma}^{\prime}(s), \nabla t(\tilde{\gamma}(s))\right) \in\left(-\frac{\pi}{2}, \frac{\pi}{2}\right), \quad s \in \mathbb{R} .
$$

then there exists a constant $\mathcal{C}(\tilde{\gamma})$ depending only on $\tilde{\gamma}$ such that

$$
f(t(\tilde{\gamma}(s))) \sin \alpha(s)=\mathcal{C}(\tilde{\gamma}), \quad s \in \mathbb{R} .
$$

We next see that the axiom of plane holds for all the warped product models as stated (see [10]).

THEOREM 2.2. (The axiom of plane for warped product models, Mashiko-Shiohama [10]) Let $(\widetilde{M}, N)$ be a warped product model and $\tilde{\gamma}:[0, a) \rightarrow \widetilde{M}$ a unit speed geodesic which is transversal to a meridian. Then $\mathcal{S}(\tilde{\gamma}) \subset \widetilde{M}$ is totally geodesic. Moreover, the inner distance of $\mathcal{S}(\tilde{\gamma})$ coincides with that of $\tilde{M}$ if $\tilde{\gamma}$ is minimizing. Here $\mathcal{S}(\tilde{\gamma})$ is the ruled surface consisting of all the meridians passing through points on $\tilde{\gamma}[0, a)$.

We finally introduce the Toponogov triangle comparison theorem for generalized geodesic triangles on $(\widetilde{M}, N)$. We assume that $(M, N)$ is referred to $(\widetilde{M}, N)$. A generalized geodesic triangle $\triangle(N x y) \subset M$ is defined by a triple of minimizing geodesics $\alpha, \beta, \gamma:[0,1] \rightarrow M$ such that

$$
\dot{\alpha}(0), \dot{\beta}(0) \in N^{\perp}, \quad \alpha(1)=\gamma(1)=y, \quad \beta(1)=\gamma(0)=x .
$$

Here $x, y \in M \backslash N$ are taken in the same component of $M \backslash N$ and $\alpha, \beta$ are minimizing geodesics from $N$. A $\triangle(N x y)$ is called a generalized narrow triangle if and only if $\alpha(t) \in B(\beta(t), \delta(M)), t \in[0,1]$. Here $\delta(M)$ is the convexity radius of $M$. The following theorem has been established in [9] and valid for pointed manifolds referred to model surfaces of revolution.

THEOREM 2.3. (Narrow triangle comparison theorem, [9]) Assume that $(M, N)$ is referred to $(\tilde{M}, N)$. Assume further that a generalized narrow triangle $\triangle(N x y) \subset M$ admits the corresponding generalized narrow triangle $\triangle(N \tilde{x} \tilde{y}) \subset \widetilde{M}$ such that

$$
d(N, x)=d(N, \tilde{x}), \quad d(N, y)=d(N, \tilde{y}), \quad d(x, y)=d(\tilde{x}, \tilde{y}) .
$$

Then we have

$$
\angle N x y \geq N \tilde{x} \tilde{y}, \quad \angle N y x \geq N \tilde{y} \tilde{x} .
$$

Note that the existence of the corresponding generalized narrow triangle in $\widetilde{M}$ is ensured by the Berger comparison theorem for focal point distance. 
Remark. Theorem 2.3 is valid for $(M, o)$ referred to a model surface $(\tilde{M}, \tilde{o})$ of revolution.

\section{Proofs of the theorems}

The crucial point of the proofs of our theorems is to verify that if $(M, o)($ or $(M, N))$ is referred to $(\tilde{M}, o)$ (or $(\tilde{M}, N))$ then $M$ can be decomposed into two disks. This is achieved by showing that the distance function to $o$ (or to $N$ ) has exactly two critical points.

Proposition 3.1. Under the same assumptions as in Theorem A, the distance function $\left.\rho_{o}\right|_{M \backslash B(o, \varepsilon(\tilde{M}))}$ to the base point o has exactly one critical point at $o_{1} \in M$, where $\rho_{o}\left(o_{1}\right)=d\left(o, o_{1}\right)=\max \rho_{o}$ and the maximum of $\rho_{o}$ is attained at a unique point.

Proof. Step 1. Suppose that $o_{1}$ and $o_{2}$ are two points in $M$ such that $\rho_{o}\left(o_{1}\right)=$ $\rho_{o}\left(o_{2}\right)=\max \rho_{o}=\ell$; that is, $o_{1}, o_{2}$ are critical points of $\rho_{o}$. Let $\sigma:[0,1] \rightarrow M$ be a minimizing geodesic in $M$ with $\sigma(0)=o_{1}$ and $\sigma(1)=o_{2}$. There exist minimizing geodesics joining $o$ to $o_{1}$ and $o_{2}$ making angles with $\sigma$ at $o_{1}, o_{2}$ not greater than $\pi / 2$. Let $0=t_{0}<t_{1}<\cdots<t_{k}=1$ be chosen such that $\triangle\left(o \sigma\left(t_{i-1}\right) \sigma\left(t_{i}\right)\right)$ for each $i=1,2, \ldots, k$ is a narrow triangle and such that $d\left(o, \sigma\left(t_{j}\right)\right)=\min _{0 \leq t \leq 1}\left(\rho_{o} \circ \sigma\right)$ for some $j(1 \leq j \leq k-1)$. From now on, we put $x_{i}:=\sigma\left(t_{i}\right)$ and $\sigma_{i}(t):=\left.\sigma\right|_{\left[t_{i}, t_{i+1}\right]}(t)$. Since $\triangle_{i-1}:=\triangle\left(o x_{i-1} x_{i}\right) \subset M$ is a narrow triangle, it admits the corresponding triangle in $\tilde{M}, \widetilde{\triangle}_{i-1}:=\triangle\left(\tilde{o} \tilde{x}_{i-1} \tilde{x}_{i}\right) \subset \tilde{M}$, satisfying (2.1) and (2.2) in Theorem 2.3. From the assumption $\ell>\tilde{\ell}-\varepsilon(\tilde{M})$, we see that $\tilde{o}_{1}$, $\tilde{o}_{2}$ exist in $B\left(o^{*}, \varepsilon(\tilde{M})\right)$. Here $o^{*} \in \tilde{M}$ is the unique point which is the farthest from $\tilde{o}$ and $d\left(\tilde{o}, o^{*}\right)=\tilde{\ell}$. If $\tilde{\sigma}_{i-1}:[0,1] \rightarrow \tilde{M}$ is the edge of $\widetilde{\triangle}_{i-1}=\triangle\left(\tilde{o} \tilde{x}_{i-1} \tilde{x}_{i}\right)$ with $\tilde{\sigma}_{i-1}(0)=\tilde{x}_{i-1}$ and $\tilde{\sigma}_{i-1}(1)=\tilde{x}_{i}$, then the Clairaut relation implies that

$$
\mathcal{C}\left(\tilde{\sigma}_{0}\right) \geq \mathcal{C}\left(\tilde{\sigma}_{1}\right) \geq \cdots \geq \mathcal{C}\left(\tilde{\sigma}_{j-1}\right) .
$$

The equality holds if and only if $\angle \tilde{x}_{i-1} \tilde{x}_{i} \tilde{x}_{i+1}=\pi$. It follows from the assumption $\rho_{o}\left(o_{1}\right)=\ell>\tilde{\ell}-\varepsilon(\tilde{M})$ and $L \tilde{o} \tilde{o}_{1} \tilde{x}_{1} \leq \pi / 2$ that

$$
\begin{aligned}
\mathcal{C}\left(\tilde{\sigma}_{0}\right)=f\left(d\left(o, o_{1}\right)\right) \sin \angle \tilde{o} \tilde{o}_{1} \tilde{x}_{1} & \leq f(\tilde{\ell}-\varepsilon(\tilde{M})) \sin \angle \tilde{o} \tilde{o}_{1} \tilde{x}_{1} \\
& \leq f(\tilde{\ell}-\varepsilon(\tilde{M})) .
\end{aligned}
$$

On the other hand, from Toponogov's theorem we get $L \tilde{o} \tilde{x}_{j} \tilde{x}_{j-1} \leq \pi / 2$. If we suppose that $L \tilde{o} \tilde{x}_{j} \tilde{x}_{j-1}<\pi / 2$ then we have a contradiction for $d\left(o, x_{j}\right)=\min \left(\rho_{o} \circ \sigma\right)$ since 
$d\left(o, x_{j}\right)=d\left(\tilde{o}, \tilde{x}_{j}\right)>d\left(\tilde{o}, \tilde{x}_{j-1}\right)=d\left(o, x_{j-1}\right)$. Thus, we see that $L \tilde{o} \tilde{x}_{j} \tilde{x}_{j-1}=\pi / 2$. From this, we get $\mathcal{C}\left(\tilde{\sigma}_{j-1}\right)=f\left(d\left(o, x_{j}\right)\right) \sin \angle \tilde{o} \tilde{x}_{j} \tilde{x}_{j-1}=f\left(d\left(o, x_{j}\right)\right)$. Therefore, we observe that $\tilde{x}_{j} \in B(\tilde{o}, \varepsilon(\tilde{M})) \cup B\left(o^{*}, \varepsilon(\tilde{M})\right)$. This follows from the choice of $\varepsilon_{1}(\tilde{M})$ and $\varepsilon(\widetilde{M})$.

First of all, we suppose that $\tilde{x}_{j} \in B(\tilde{o}, \varepsilon(\tilde{M}))$. A contradiction is derived by the triangle inequality:

$$
\begin{aligned}
\sum_{i=0}^{k-1} L\left(\tilde{\sigma}_{i}\right) & =\sum_{i=0}^{j-1} L\left(\tilde{\sigma}_{i}\right)+\sum_{i=j}^{k-1} L\left(\tilde{\sigma}_{i}\right) \\
& \geq\left\{d\left(\tilde{o}, \tilde{o}_{1}\right)-d\left(\tilde{o}, \tilde{x}_{j}\right)\right\}+\left\{d\left(\tilde{o}, \tilde{o}_{2}\right)-d\left(\tilde{o}, \tilde{x}_{j}\right)\right\} \\
& >2(\tilde{\ell}-2 \varepsilon(\tilde{M})) .
\end{aligned}
$$

On the other hand,

$$
\sum_{i=0}^{k-1} L\left(\tilde{\sigma}_{i}\right)=\sum_{i=0}^{k-1} L\left(\sigma_{i}\right)=d\left(o_{1}, o_{2}\right) \leq \operatorname{diam}(M) \leq \tilde{\ell}
$$

This means that $\tilde{x}_{j} \in B\left(o^{*}, \varepsilon(\tilde{M})\right)$. Since $d\left(o, x_{j}\right)=d\left(\tilde{o}, \tilde{x}_{j}\right)=\min \left(\rho_{o} \circ \sigma\right)$, we can think about the following two cases.

First, we consider the case $d\left(o, x_{j}\right)=\ell$; that is, the distance function $\left(\rho_{o} \circ \sigma\right)$ : $[0,1] \rightarrow \mathbb{R}_{+}$is constant. Then the narrow triangle $\triangle_{j-1}=\Delta\left(o_{j-1} x_{j}\right) \subset$ $M$ is an isosceles triangle and $L o x_{j} x_{j-1}=\pi / 2$. By Toponogov's theorem, $\widetilde{\triangle}_{j-1}=\triangle\left(\tilde{o} \tilde{x}_{j-1} \tilde{x}_{j}\right) \subset \tilde{M}$ is also an isosceles triangle and $L \tilde{o} \tilde{x}_{j} \tilde{x}_{j-1} \leq \pi / 2$. Moreover, we see that $\triangle\left(o^{*} \tilde{x}_{j-1} \tilde{x}_{j}\right) \subset \tilde{M}$ is an isosceles triangle with $d\left(o^{*}, \tilde{x}_{j-1}\right)=$ $d\left(o^{*}, \tilde{x}_{j}\right)$. Since $B\left(o^{*}, \varepsilon(\tilde{M})\right)$ is convex ball and two points $\tilde{x}_{j-1}, \tilde{x}_{j}$ are contained in $B\left(o^{*}, \varepsilon(\tilde{M})\right)$, we see $\angle o^{*} \tilde{x}_{j} \tilde{x}_{j-1}<\pi / 2$. Further $\angle \tilde{o} \tilde{x}_{j} \tilde{x}_{j-1}>\pi / 2$. This is a contradiction.

Next, we suppose that $d\left(o, x_{j}\right)<\ell$. For the narrow triangle $\triangle_{j-1}=\triangle\left(o x_{j-1} x_{j}\right)$ in $M$, there exists the corresponding narrow triangle $\widetilde{\triangle}_{j-1}=\triangle\left(\tilde{o} \tilde{x}_{j-1} \tilde{x}_{j}\right)$ in $\widetilde{M}$ with $d\left(\tilde{o}, \tilde{x}_{j}\right)=d\left(o, x_{j}\right)<d\left(\tilde{o}, \tilde{x}_{j-1}\right)=d\left(o, x_{j-1}\right)$. This implies that $L \tilde{o} \tilde{x}_{j} \tilde{x}_{j-1}>\pi / 2$, a contradiction to the choice of $x_{j}$.

Step 2. Next we show that there is no critical point $q$ of $\rho_{o}$ satisfying $\varepsilon(\tilde{M}) \leq$ $\rho_{o}(q)<\max \rho_{o}$. We suppose that there exists a critical point $q \in M$ such that $\varepsilon(\tilde{M}) \leq d(o, q)<\ell$. For a minimizing geodesic $\sigma:[0,1] \rightarrow M$ with $\sigma(0)=$ $o_{1}$ and $\sigma(1)=q$, there exist minimizing geodesics $\gamma_{1}, \gamma_{2}:[0,1] \rightarrow M$ with $\gamma_{1}(0)=o=\gamma_{2}(0), \gamma_{1}(1)=q, \gamma_{2}(1)=o_{1}$ such that $L\left(-\dot{\gamma}_{1}(1),-\dot{\sigma}(1)\right) \leq \pi / 2$ and $L\left(-\dot{\gamma}_{2}(1), \dot{\sigma}(0)\right) \leq \pi / 2$. Choose a partition $0=t_{0}<t_{1}<\cdots<t_{k}=1$ 
such that $\triangle\left(o \sigma\left(t_{i-1}\right) \sigma\left(t_{i}\right)\right)$ for every $i=1, \ldots, k$ is a narrow triangle and such that $d\left(o, \sigma\left(t_{j}\right)\right)=\min \left(\rho_{o} \circ \sigma\right)$ for some $0<j<k$. Put $x_{i}:=\sigma\left(t_{i}\right)$ and $\sigma_{i}:=\left.\sigma\right|_{\left[t_{i}, t_{i+1}\right]}$ for each $i$. Since $\triangle_{i-1}:=\triangle\left(o x_{i-1} x_{i}\right)$ is a narrow triangle in $M$, it admits the corresponding triangle $\widetilde{\triangle}_{i-1}:=\triangle\left(\tilde{o} \tilde{x}_{i-1} \tilde{x}_{i}\right) \subset \tilde{M}$ satisfying (2.1) and (2.2). If $\tilde{\sigma}_{i-1}:[0,1] \rightarrow \widetilde{M}$ is the edge of $\widetilde{\triangle}_{i-1}$ with $\tilde{\sigma}_{i-1}(0)=\tilde{x}_{i-1}$ and $\tilde{\sigma}_{i-1}(1)=\tilde{x}_{i}$, then the Clairaut relation implies that

$$
\mathcal{C}\left(\tilde{\sigma}_{0}\right) \geq \mathcal{C}\left(\tilde{\sigma}_{1}\right) \geq \cdots \geq \mathcal{C}\left(\tilde{\sigma}_{j-1}\right)
$$

and

$$
\mathcal{C}\left(\tilde{\sigma}_{k-1}\right) \geq \mathcal{C}\left(\tilde{\sigma}_{k-2}\right) \geq \cdots \geq \mathcal{C}\left(\tilde{\sigma}_{j}\right) .
$$

Here the equality holds if and only if $\angle \tilde{x}_{i-1} \tilde{x}_{i} \tilde{x}_{i+1}=\pi$. From (3.1) and Toponogov's theorem, we see that $\tilde{x}_{j} \in B(\tilde{o}, \varepsilon(\tilde{M})) \cup B\left(o^{*}, \varepsilon(\tilde{M})\right)$.

Firstly, we suppose $\tilde{x}_{j} \in B(\tilde{o}, \varepsilon(\tilde{M}))$. Since $q \notin B(o, \delta(o))$,

$$
\begin{aligned}
\sum_{i=0}^{k-1} L\left(\tilde{\sigma}_{i}\right) & =\sum_{i=0}^{j-1} L\left(\tilde{\sigma}_{i}\right)+\sum_{i=j}^{k-1} L\left(\tilde{\sigma}_{i}\right) \\
& \geq\left\{d\left(\tilde{o}, \tilde{o}_{1}\right)-d\left(\tilde{o}, \tilde{x}_{j}\right)\right\}+\left\{d(\tilde{o}, \tilde{q})-d\left(\tilde{o}, \tilde{x}_{j}\right)\right\} \\
& >\tilde{\ell}-3 \varepsilon(\tilde{M})+3 \varepsilon_{1}(\tilde{M}) .
\end{aligned}
$$

On the other hand,

$$
\sum_{i=0}^{k-1} L\left(\tilde{\sigma}_{i}\right)=\sum_{i=0}^{k-1} L\left(\sigma_{i}\right)=d\left(o_{1}, q\right) \leq \operatorname{diam}(M) \leq \tilde{\ell} .
$$

From these inequalities and the assumption $\varepsilon(\tilde{M}) \leq \varepsilon_{1}(\tilde{M})$, we have a contradiction.

Next, we suppose that $\tilde{x}_{j} \in B\left(o^{*}, \varepsilon(\tilde{M})\right)$. Since the point $x_{j}$ is realized at a distance from the base point $o$ to the minimizing geodesic $\sigma$ (that is, $d\left(o, x_{j}\right)=$ $\left.\min _{t \in[0,1]}\left(\rho_{o} \circ \sigma\right)(t)\right)$, we have $d\left(\tilde{o}, \tilde{x}_{j}\right)<\ell$. Also, since $B\left(o^{*}, \varepsilon(\tilde{M})\right)$ is convex ball, $L \tilde{o} \tilde{x}_{j} \tilde{o}_{1}>\pi / 2$.

Therefore, we prove the uniqueness of critical point $o_{1} \in M \backslash B(o, \varepsilon(\tilde{M}))$ of $\left.\rho_{o}\right|_{M \backslash B(o, \varepsilon(\tilde{M}))}$ such that $d\left(o, o_{1}\right)=\max \rho_{o}$.

Next, we prove Theorem B. The method of the proof is similar to that of Theorem A. The following proposition is proved by a similar manner as stated in the proof of Proposition 3.1 and omitted here.

Proposition 3.2. Let $(\tilde{M}, N)$ be the warped product $\mathbf{S}^{n}$-models. Let $(M, N)$ be referred to $(\tilde{M}, N)$. Under the assumption $\ell_{+}>\tilde{\ell}_{+}-\eta_{+}(\tilde{M}), M_{+}$is a topological disk. 
Remark. We can obtain the same consequence to $M_{-}$by using a method similar to that which is used in the above proposition. From this, we see that $(M, N)$ is composed with two topological disks. Therefore, we have proved that $(M, N)$ is homeomorphic to $\mathbf{S}^{n}$.

Acknowledgements. The author would like to thank Professor K. Shiohama for suggesting these theorems and for his kind encouragement.

\title{
REFERENCES
}

[1] U. Abresch. Lower curvature bounds, Toponogov's theorem and bounded topology I. Ann. Sci. ecole Norm. Sup. 18 (1985), 651-670.

[2] U. Abresch. Lower curvature bounds, Toponogov's theorem and bounded topology II. Ann. Sci. ecole Norm. Sup. 20 (1987), 475-502.

[3] D. Elerath. An improved Toponogov comparision theorem for nonnegatively curved manifolds. J. Diff. Geom. 15 (1980), 187-216.

[4] R. E. Greene and H. Wu. Function Theory on Manifolds Whose Posses a Pole (Lecture Notes in Mathematics, 699). Springer, Berlin, 1979.

[5] K. Kondo. The topology of complete manifolds with radial curvature bounded below. Preprint, 2003.

[6] K. Kondo. A radius sphere theorem for manifolds with radial curvature below. Preprint, 2003.

[7] Y. Machigashira. Manifolds with pinched radial curvature. Proc. Amer. Math. Soc. 118 (1993), 979-985.

[8] Y. Machigashira. Complete open manifolds of non-negative radial curvature. Pacific J. Math. 165 (1994), 153-160.

[9] Y. Mashiko and K. Shiohama. Comparison geometry referred to the warped product models. Tohoku Math. J. to appear.

[10] Y. Mashiko and K. Shiohama. The axiom of plane on warped product models and its application. Kyushu J. Math. 59 (2005), 385-392.

[11] Y. Machigashira and K. Shiohama. Riemannian manifolds with positive radial curvature. Japan. J. Math. 19 (1993), 419-430.

[12] V. B. Marenich. Manifolds with minimal radial curvature bounded form below and big volume. Trans. Amer. Math. Soc. 352 (2000), 4451-4468.

[13] V. B. Marenich and S. J. X. Medconça. Manifolds with minimal radial curvature bounded from below and big radius. Indiana Univ. Math. J. 48 (1999), 249-274.

[14] K. Shiohama. A sphere theorem for manifolds with positive Ricci curvature. Trans. Amer. Math. Soc. 275 (1983), 811-819.

\author{
Hyunjin Lee \\ Department of Mathematics \\ Faculty of Science and Engineering \\ Saga University \\ Honjyo-machi \\ Saga 840-8502, Japan \\ (E-mail:leehj@ms.saga-u.ac.jp)
}

